\title{
Spin-wave wells revisited: From wavelength conversion and Möbius modes to magnon valleytronics
}

\author{
K. G. Fripp and V. V. Kruglyak $๑^{*}$ \\ University of Exeter, Stocker Road, Exeter EX4 4QL, United Kingdom
}

(Received 11 January 2021; accepted 20 April 2021; published 3 May 2021)

\begin{abstract}
We have used micromagnetic simulations to model backward-volume dipole-exchange spin waves in graded profiles of the bias magnetic field. We demonstrate spin-wave wavelength conversion upon the wave's reflection from turning points due to the two characteristic minima (" $U$ points") occurring in their dispersion at finite (nonzero) wave vectors. As a result, backward-volume dipole-exchange spin waves confined in "spin-wave wells" either form Möbius modes, making multiple real-space turns for each reciprocal-space round trip, or split into pairs of degenerate modes in the valleys near the two $U$ points. The latter modes may therefore be assigned a pseudospin. We show that the pseudospin can be switched by scattering the spin wave from decreases of the bias magnetic field, while it is immune to scattering from field increases. Pseudospin creation and read-out can be accomplished using chiral spin-wave transducers, as described in Au et al. [Appl. Phys. Lett. 100, 182404 (2012)] and Au et al. [Appl. Phys. Lett. 100, 172408 (2012)], respectively. Taken together, the possibility of pseudospin creation, manipulation, and read-out suggests a path to development of a spin-wave version of valleytronics ("magnon valleytronics"), in which the pseudospin (rather than amplitude or phase) of spin waves would be used to encode data. Our results are not limited to graded bias magnetic field but can be generalized to other magnonic media with spatially varying characteristics, produced using the toolbox of graded index magnonics.
\end{abstract}

DOI: 10.1103/PhysRevB.103.184403

\section{INTRODUCTION AND BACKGROUND}

The phenomena of wave propagation, scattering, and quantization play the pivotal role in both classical [1] and quantum [2] physics. In the one-dimensional (1D) case, the quantization is observed when a wave is confined between two barriers, causing the wave to be backscattered. When the barriers are smoothly graded rather than sharp (i.e., have properties slowly rather than abruptly varying in space), the wave does not "feel" the magnetic gradients until it reaches the turning points, which causes its backscattering. Beyond the turning points, the wave's propagation is forbidden (for the given frequency): The wave number turns imaginary, and so, the wave decays exponentially in space. At the turning point itself, the wave number is (usually) equal to zero, which corresponds to the " $\Gamma$ point" in the reciprocal space. Consequently, the wave numbers of the incident and backscattered waves have opposite signs. This typical scenario is realized, e.g., for light, sound, and quantum-mechanical particles.

Very different behavior is expected for waves whose dispersion relation has frequency minima at finite (nonzero) wave vectors. Such a dispersion relation is found, e.g., for rotons in liquid helium [3-6], for electrons in graphene near the Fermi level [7-10], and for dipole-exchange spin waves-wavelike excitations of the magnetization in magnetically ordered materials [11-13] - in the backward-volume dipole-exchange spin-wave (BVDESW) geometry [12,14]. The propagation, backscattering, and confinement of this latter type of spin wave is studied theoretically in this paper.

\footnotetext{
*V.V.Kruglyak@exeter.ac.uk
}

Their dispersion, plotted in Fig. 1, can have two "valleys" around frequency minima at finite wave numbers (which we call " $U$ points"). Importantly, such spin waves can be not only modeled numerically (as we do here) but also detected and imaged experimentally $[15,16]$. This promises a direct experimental verification of the effects predicted in this paper-backscattering-induced wavelength conversion and Möbius-like confinement-and gives credibility to the proposed development of a spin-wave version of valleytronics ("magnon valleytronics").

As an illustration, let us consider a BVDESW wave packet with central frequency $f$. The wave packet propagates in nonuniform bias magnetic field $H(x)$ that varies smoothly with coordinate $x$. In the commonly used Wentzel-KramersBrillouin (WKB) approximation [2], applied to spin waves, e.g., in Refs. [17-26], it is assumed that the wave's dispersion relation, which may be written for BVDESWs as [14]

$$
\begin{aligned}
& F(k, H) \\
& \quad=\mu_{0} \gamma \sqrt{\left(H+\frac{2 A}{\mu_{0} M} k^{2}\right)\left[H+\frac{2 A}{\mu_{0} M} k^{2}+M \frac{1-\exp (-k d)}{k d}\right]},
\end{aligned}
$$

may still be introduced and is satisfied for each value of $x$. Then, the packet's central wave number $k(x)$ follows the field variation adiabatically, while the value of $f$ is conserved [27]:

$$
f=F[k(x, f), H(x)]=\text { const. }
$$

The discrete frequencies $f_{n}$ of modes confined in regions of reduced magnetic field can be calculated using the Bohr- 
Sommerfeld quantization formula [2],

$$
\oint k\left(x, f_{n}\right) d x=2 \pi n,
$$

where $n=1,2,3, \cdots$, and $k\left(x, f_{n}\right)$ is found from Eq. (2). The integration is performed along the wave packet's closed trajectory, yielding the area enclosed by the trajectory in the phase space. Usually, Eq. (3) is equivalent to

$$
\int_{x_{0}}^{x_{1}} k(x) d x=\pi n
$$

where $x_{0}$ and $x_{1}$ are the coordinates of the two turning points between which the wave is confined. In Refs. [19,20,2224,26], Eq. (4) was also applied to the analysis of the quantization of BVDESWs. The spin waves were confined in the demagnetized regions ("spin-wave wells") near the edges of patterned magnetic elements (e.g., stripes) orthogonal to the direction of the applied magnetic field. However, the use of Eq. (4) instead of Eq. (3) could only be justified if the wavelength of the spin waves was preserved upon backscattering from the turning points, which was not verified in Refs. $[19,20-24,26]$ and is therefore done here.

The paper is organized as follows. Section II is devoted to the methods used in our numerical micromagnetic simulations and data analyses. In Sec. III, we describe our results, focusing on the BVDESW confinement and wavelength conversion in different field profiles, and on the concepts of BVDESW pseudospin and magnon valleytronics. Section IV contains a qualitative discussion of the implications of our findings for the use of the Bohr-Sommerfeld quantization formulas for BVDESWs. In Sec. V, we summarize the implications of our findings to various other research directions in magnonics.

\section{METHODS}

We simulate the scattering and confinement of BVDESW wave packets in $1 \mathrm{D}$ profiles of the bias magnetic field using the MUMAX3 software [28]. The magnonic medium has permalloylike properties: the saturation magnetization of 800 $\mathrm{kA} / \mathrm{m}$, the exchange stiffness of $13 \mathrm{pJ} / \mathrm{m}$, and zero magnetocrystalline anisotropy. To make the wave effects clearer, we assume the Gilbert damping parameter to have a very low value of 0.0001 , inherent to high-quality ferrites rather than permalloy. The samples have dimensions of $l \times 10 \mathrm{~nm} \times$ $80 \mathrm{~nm}(x \times y \times z)$, where $l$ is typically in the range of several tens of micrometers. One-dimensional periodic boundary conditions are used in the $y$ direction, to remove any in-plane shape anisotropy. The simulation volume is discretized into cuboidal cells each dimension of which is $5 \mathrm{~nm}$ or smaller. The bias magnetic field is applied in the $x$ direction, with the equilibrium magnetization configuration established prior to dynamical simulations.

All dynamic magnetic fields in our simulations here are uniform in the $y$ and $z$ directions. To calculate the spin-wave dispersion (Fig. 1), a uniform film in a uniform bias magnetic field is excited by a dynamic magnetic field the profile of which is given by the sinc function both in time (cutoff frequency $25 \mathrm{GHz}$ ) and along the $x$ direction (cutoff wave number $\pi / 20 \mathrm{rad} / \mathrm{nm}$ ) [29]. To excite BVDESW wave packets,

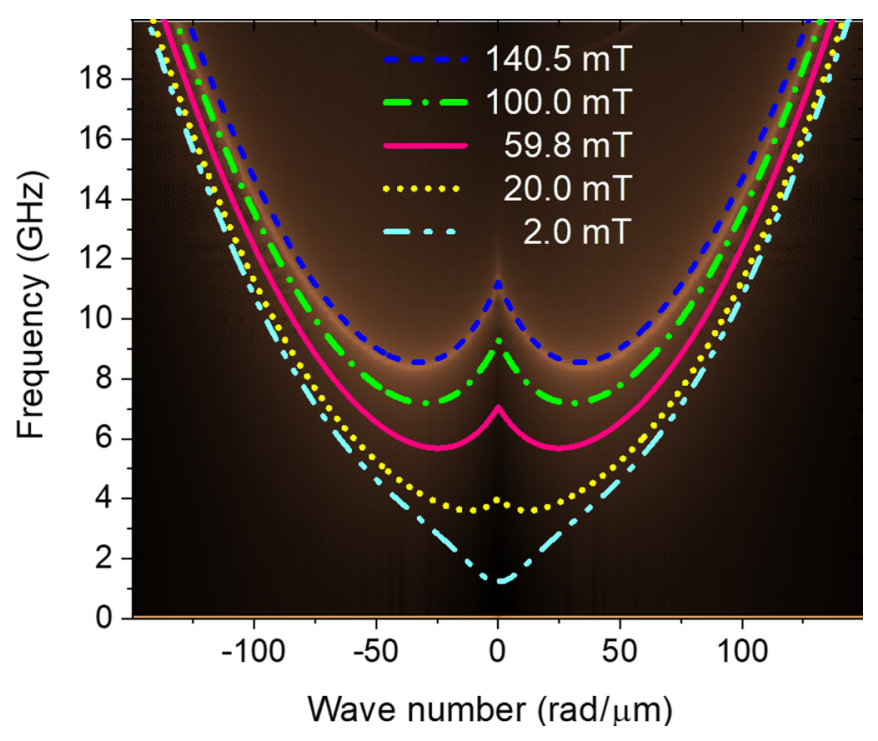

FIG. 1. The lines show the dispersion relation of BVDESWs plotted using Eq. (1) for the indicated values of the bias magnetic field. The curve for $2 \mathrm{mT}$ exemplifies the dispersion relation of "conventional waves," which has the frequency minimum at the $\Gamma$ point. The background shows the dispersion for the field of $140.5 \mathrm{mT}$ obtained by Fourier-transforming results of the micromagnetic simulations (brighter color corresponds to greater spin-wave amplitude).

we use dynamic magnetic fields with running-wave profiles [30,31]:

$$
\begin{aligned}
h= & h_{0} \exp \left[-\frac{\left(x-x_{0}\right)^{2}}{2 \sigma_{x}^{2}}\right] \exp \left[-\frac{\left(t-t_{0}\right)^{2}}{2 \sigma_{t}^{2}}\right] \\
& \times \cos \left( \pm k_{0} x-2 \pi f t\right) .
\end{aligned}
$$

Here, $h_{0} \leqslant 0.1 \mathrm{mT}$ is the maximum transient field amplitude, $f$ is the central frequency of the wave packet, and $k_{0}$ is the corresponding wave number, obtained from the preliminarily computed dispersion relation, such as the one shown in Fig. 1 . The wave packets are excited centered at position $x_{0}$ at time $t_{0}$ relative to the beginning of the simulation, to suit the goal of each particular simulation. The size and duration of the spatial and temporal envelopes of the field are defined by parameters $\sigma_{x}$ and $\sigma_{t}$, respectively. Their values are chosen so as to excite a small band of frequencies and wave numbers around the desired central values while also ensuring that the packet fits and has room for movement within the well formed by the field profile. The spin-wave well sizes of about 10 $20 \mu \mathrm{m}$ considered here represent a compromise of this sort. Very similar results are obtained for larger-scale field profiles. However, it becomes impossible to form wave packets with well-defined trajectories in wells with sizes of about $2 \mu \mathrm{m}$ or smaller, which will be addressed elsewhere. To obtain the spin-wave dispersion relations and wave-packet trajectories, we employ standard Fourier transform techniques, described, e.g., in Refs. [29-31]. 


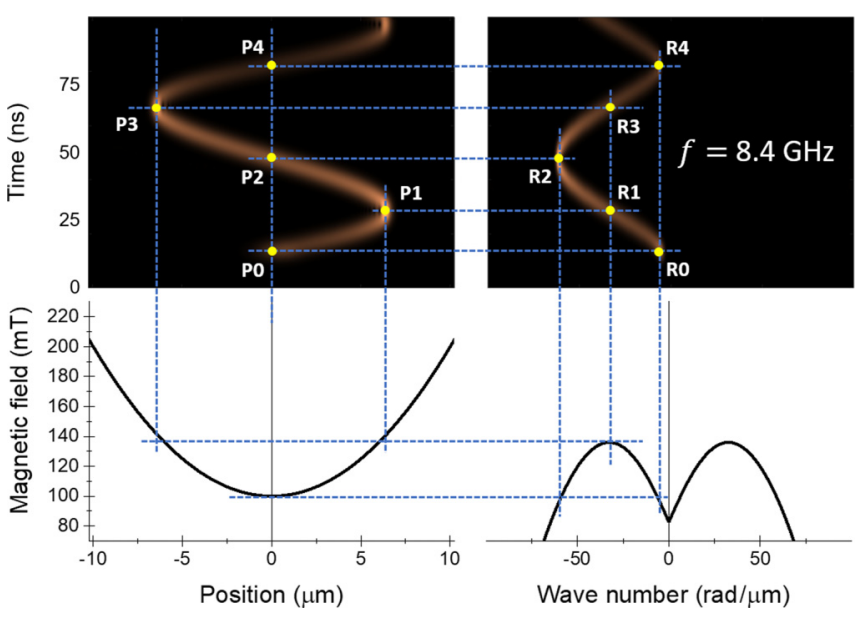

FIG. 2. The wave-packet trajectories (top row) and corresponding magnetic field profiles (bottom row) are shown in the real (left column) and reciprocal (right column) space for the central frequency of $8.4 \mathrm{GHz}$. The brighter color corresponds to greater spin-wave amplitude. The dashed lines and points at their crossings show the correspondence between features from the different panels. See the Supplemental Material [33] for an animated version of this figure.

\section{RESULTS}

\section{A. Backward-volume dipole-exchange spin-wave (BVDESW) dispersion}

Figure 1 compares the BVDESW dispersion relation computed from the results of our micromagnetic simulations with the analytical curve plotted using Eq. (1), for a uniform bias field of $0.1405 \mathrm{~T}$. The numerical and analytical results agree well at small wave numbers. The discrepancy developing for shorter wavelengths may be attributed to the approximate character of either Eq. (1) [14], or micromagnetic simulations [29], or both. For 0.1405 T, the two characteristic frequency minima (the $U$ points) correspond to the wave numbers of \pm $35 \mathrm{rad} / \mu \mathrm{m}$ and the frequency of $8.4 \mathrm{GHz}$. As the bias field is reduced, the $U$ points shift toward the $\Gamma$ point and eventually disappear.

\section{B. Symmetric spin-wave well: Low-frequency confinement}

The propagation and confinement of BVDESW wave packets differs qualitatively depending on whether the wave packet's central frequency is below or above that of the ferromagnetic resonance (FMR) at the lowest value of the bias magnetic field. Here, we consider the case of "low-frequency confinement," i.e., when the central frequency is below the FMR value but is above the $U$-point frequency at the field minimum. The top left panel in Fig. 2 shows the real-space trajectory $\mathrm{P} 0-\mathrm{P} 4$ of the envelope of a Gaussian magnonic wave packet ("wave-packet trajectory") at the central frequency of $8.4 \mathrm{GHz}$ confined in a symmetric spin-wave well. This well is created by the parabolic magnetic-field profile with a minimum of $0.1 \mathrm{~T}$, shown in the bottom left panel. The corresponding reciprocal-space trajectory R0-R4 (top right) reveals that the turning points in real $(\mathrm{P} 1, \mathrm{P} 3)$ and reciprocal (R0, R2, R4) space do not coincide: The wave packet continues moving forward in reciprocal (real) space when it is turned back in real (reciprocal) space. This is due to the difference between the group and phase velocities of spin waves, apparent from Fig. 1. The character of the trajectories can be explained in greater detail by considering the variation of the packet's central wave number with the field (bottom right). The packet begins its journey from points $\mathrm{P} 0$ and $\mathrm{R} 0$ in the real and reciprocal space, respectively, at the bias field of $0.1 \mathrm{~T}$. At this field, the frequency of $8.4 \mathrm{GHz}$ corresponds to the wave numbers of $\pm 61.5 \mathrm{rad} / \mu \mathrm{m}$ (shorter wavelength) and $\pm 4.5 \mathrm{rad} / \mu \mathrm{m}$ (longer wavelength). We launch the wave packet with the central wave number of $-4.5 \mathrm{rad} / \mu \mathrm{m}$, where the negative sign ensures that the group velocity is positive [32], so the packet propagates from left to right.

The first real-space turning point $(\mathrm{P} 1)$ corresponds to the magnetic field of about $0.1405 \mathrm{~T}$, at which the $U$ point is reached in the reciprocal space at $-35 \mathrm{rad} / \mu \mathrm{m}$ (R1). Notably, although the wave packet reverses its direction of travel in the real space, it continues to propagate in the same direction through the $U$ point in the reciprocal space. Hence, when the packet returns to the start of the journey in the real space at $\mathrm{P} 2$, its central wave number is converted from the original value of $-4.5 \mathrm{rad} / \mu \mathrm{m}(\mathrm{R} 0)$ to a new value of $-61.5 \mathrm{rad} / \mu \mathrm{m}(\mathrm{R} 2)$. As the packet propagates further, gets backscattered from the other real-space turning point at $\mathrm{P} 3$, and returns to the start again at $\mathrm{P} 4$, its central wave number is converted back to the original value of $-4.5 \mathrm{rad} / \mu \mathrm{m}$ (R4). Importantly, the wave number never switches its sign, so that the packet remains in the same valley, near the same $U$ point in the dispersion relation. This is because the frequency of $8.4 \mathrm{GHz}$ is below the FMR and above the $U$-point frequencies at the field of $0.1 \mathrm{~T}$ corresponding to the bottom of the spin-wave well. As a result, confined modes belonging to the same valley have "traveling" profiles, as discussed, e.g., in relation to dipole-exchange spin waves in obliquely magnetized films $[34,35]$.

\section{Symmetric spin-wave well: High-frequency confinement and Möbius modes}

Figure 3 shows wave-packet trajectories for the case when its central frequency of $10.5 \mathrm{GHz}$ exceeds the FMR value at the bottom of the well. Then, the wave packet is not restricted to any valley. To ensure confinement within a real-space region similar to that in Fig. 2, the parabolic spin-wave well has steeper walls. Also, for the sake of illustration, we launch a wave packet of short, exchange dominated spin waves: i.e., a wave packet with a wave number of $82 \mathrm{rad} / \mu \mathrm{m}$ (R0) is excited at point $\mathrm{P} 0$. The shape of the reciprocal-space trajectory R0-R8 is qualitatively similar to that in Fig. 2. However, the real-space trajectory $\mathrm{P} 0-\mathrm{P} 8$ changes drastically. Upon the first backscattering from the "high-field" turning point at $\mathrm{P} 1$, the wave packet propagates through the $U$ point (R1); its wavelength continues to increase as the magnetic field decreases; and once the $\Gamma$ point is reached (R2), the wave packet is backscattered at P2 [36-41]. The latter "low-field" turning point occurs even before the wave packet reaches the bottom of the spin-wave well in the real space. In the reciprocal space, unlike transitions through the $U$ points at high-field values, this transition through the $\Gamma$ point leads to switching of the valley pseudospin. When the central frequency exceeds the 


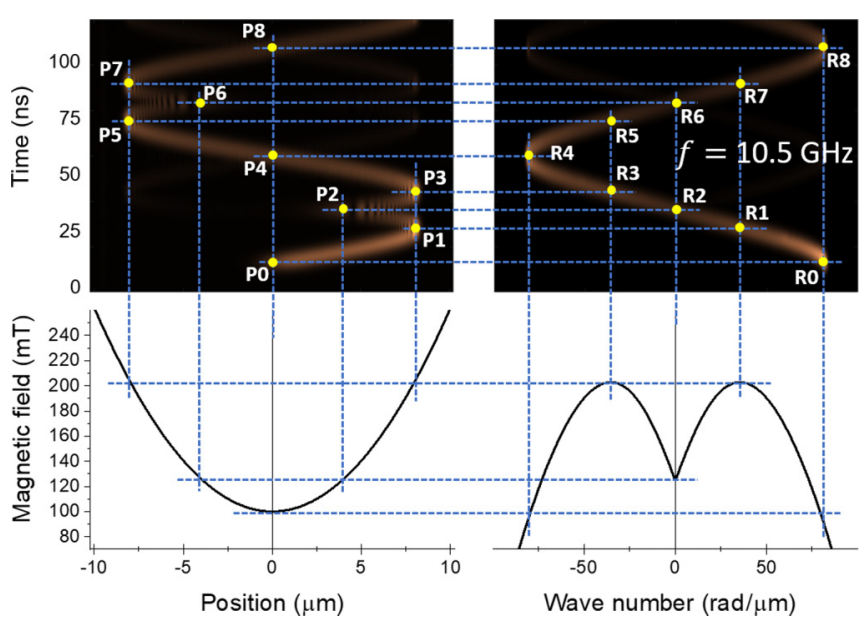

FIG. 3. The wave-packet trajectories (top row) and corresponding magnetic field profiles (bottom row) are shown in the real (left column) and reciprocal (right column) space for the central frequency of $10.5 \mathrm{GHz}$. The brighter color corresponds to greater spin-wave amplitude. The dashed lines and points at their crossings show the correspondence between features from the different panels. See the Supplemental Material [33] for an animated version of this figure.

FMR value by a smaller amount, a fraction of the wave packet tunnel through the bottom of the well [36-41] (not shown). Upon another backscattering from the same high-field turning point at $\mathrm{P} 3$, the wave packet propagates through the other $U$ point (R3), and its wavelength continues to decrease (rather than to increase) as the magnetic field decreases. Therefore, in contrast to its long-wavelength counterpart (P2, R2), the short-wavelength packet propagates through the bottom of the well without any scattering $(\mathrm{P} 4)$. As the field begins to increase, the wavelength also begins to increase (R4), and the whole scenario repeats itself in the left half of the spin-wave well (P4-P8, R4-R8). Overall, the wave packet changes its direction of travel in the real space six times (P1, P2, P3, P5, $\mathrm{P} 6$, and P7) during just a single round trip in the reciprocal space. In Ref. [42], nonlinear spin waves needing to make two real-space round trips to meet the initial phase condition were tagged "Möbius solitons." The behavior of linear confined modes exemplified in Fig. 3 is more complex than that in Ref. [42]. However, this behavior justifies such spin waves to be called "Möbius modes."

\section{Möbius modes in an asymmetric spin-wave well}

Figure 4 presents BVDESW wave-packet trajectories in the real (P0-P9, top left) and reciprocal (R0-R9, top right) space obtained from simulations with an antisymmetric, slopy magnetic field profile (bottom left). This profile is qualitatively similar to the one from Refs. [19-24]: the wave packet is confined by the field increase and the physical edge of the magnetic sample on the right- and left-hand sides, respectively. The region of nearly constant field around $x=0$ is introduced to simplify the excitation of regular Gaussian wave packets. The wave packet has a central frequency of $8.3 \mathrm{GHz}$ and central wave number of $-3 \mathrm{rad} / \mu \mathrm{m}$ at the bias field of $0.1 \mathrm{~T}(\mathrm{P} 0, \mathrm{R} 0)$. In the real space, the wave packet propagates

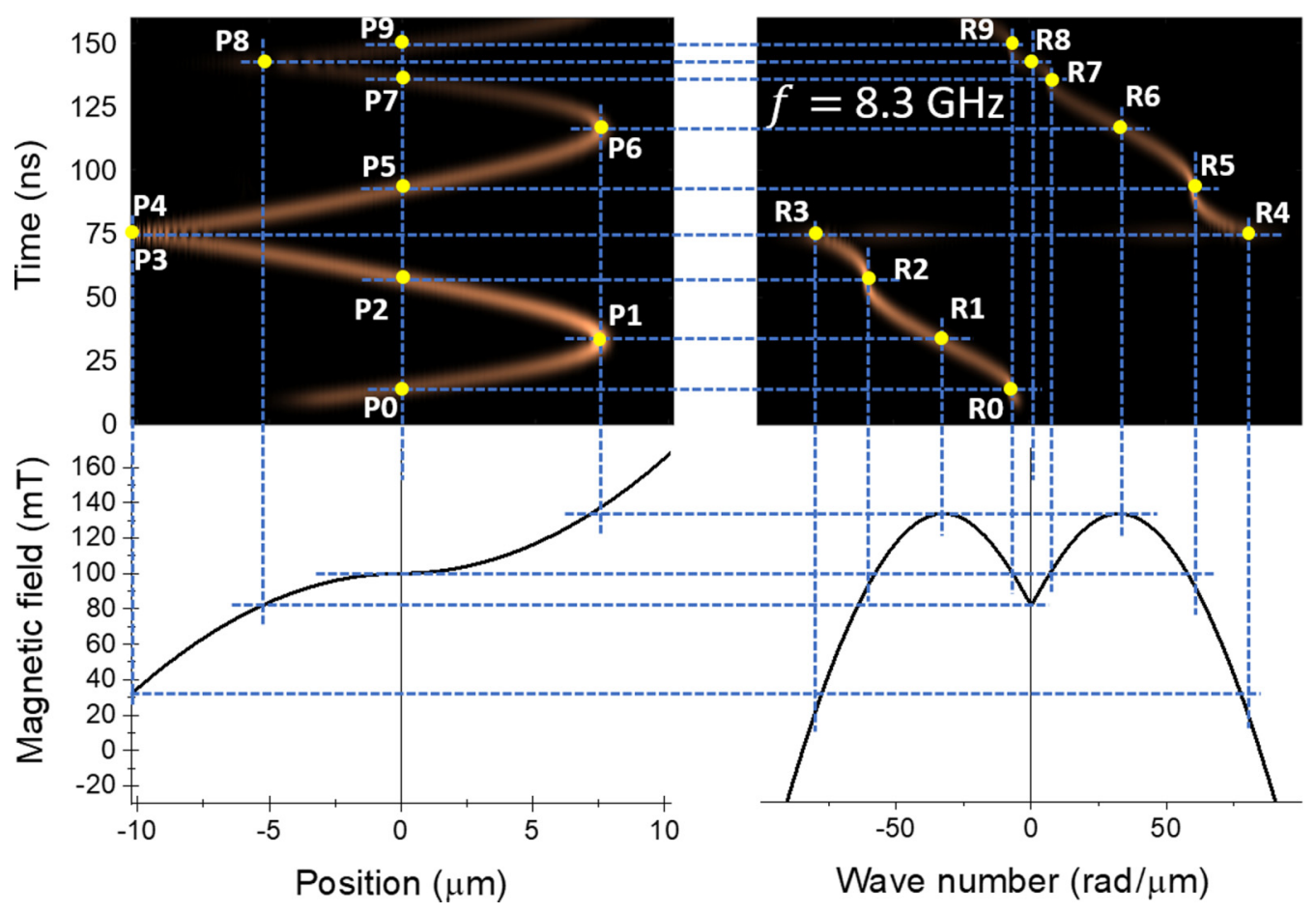

FIG. 4. The wave-packet trajectories (top row) and corresponding magnetic field profiles (bottom row) are shown in the real (left column) and reciprocal (right column) space for the frequency of $8.3 \mathrm{GHz}$. The brighter color corresponds to greater spin-wave amplitude. The dashed lines and points at their crossings show the correspondence between features from the different panels. See the Supplemental Material [33] for an animated version of this figure. 
to the right, where it encounters the parabolic field increase and is eventually backscattered (P1). In the reciprocal space, the packet continues its journey in the same direction through point R1 and then R2, so that it returns to its real-space start position (P2) with a much-shortened wavelength. Then, it bounces back from the physical edge of the sample (point $\mathrm{P} 3 / \mathrm{P} 4$ ), changing the sign but retaining the absolute value of the central wave number, which corresponds to a jump from R3 to R4 in the reciprocal space. This jump is followed by a one-directional evolution of the wave number (R4-R9). During this, the wave packet experiences two real-space reflections: from the high-field (P6) and low-field (P8) turning points, corresponding to a $U$ point (R6) and the $\Gamma$ point (R8), respectively. Overall, the wave packet changes its real-space direction of travel four times (P1, P3/P4, P6, and P8) during a single round trip in the reciprocal space.

\section{E. Magnon valleytronics}

The results shown in Fig. 2 suggest that BVDESWs with frequencies below the FMR and above the $U$-point frequencies could be used to develop a spin-wave version of "valleytronics"- a paradigm exploiting the wave's affiliation with a particular valley as an additional degree of freedom, i.e., as a "pseudospin" [7]. Inspecting Fig. 1, we see that the different BVDESW pseudospins can be easily distinguished. Indeed, at each frequency, copropagating modes (i.e., modes with group velocities of equal sign) of opposite pseudospin (i.e., from the different valleys) have different wavelengths, while counterpropagating modes of the same wavelength have opposite pseudospins. Also, the pseudospin can be associated with the sign of the phase velocity (wave number), which remains the same (irrespective of the group velocity) for each valley mode. Finally, the traveling profiles inherent to the confined modes with a particular pseudospin could be interpreted as spin current $[34,35]$. The wave number locked, chiral resonant microwave to spin-wave transducers from Ref. [43] and spin-wave valves (or phase shifters) from Ref. [44] could be used to create and to read out, respectively, magnonic states depending on their pseudospin [43-45].

To substantiate the claim of valleytronics, one must also demonstrate how the pseudospin can be "switched." Figure 5 shows results of simulations in which a BVDESW wave packet is incident on a region of reduced bias magnetic field. Panel (a) shows the field profile, while the trajectories from rows (b) and (c) correspond to wave packets of opposite pseudospin. The long wavelength/negative phase velocity wave packet [Fig. 5(b)] partly tunnels through and partly is reflected from the field decrease, as reported earlier [36-41]. The tunneled part of the wave packet retains its pseudospin. In contrast, the packet's reflected portion goes through the $\Gamma$ point and therefore switches its pseudospin. As an aside, we note that the same pseudospin switching event occurs also at points P2/R2 and P6/R6 in Fig. 3 and at point P8/R8 in Fig. 4. The short wavelength/positive phase velocity wave packet [Fig. 5(c)] passes through the field decrease ballistically, experiencing negligible reflection, if any, and retains its pseudospin. Similar simulations for scattering from regions of increased bias magnetic field (not shown) have revealed that the pseudospin is immune to both backscatter-

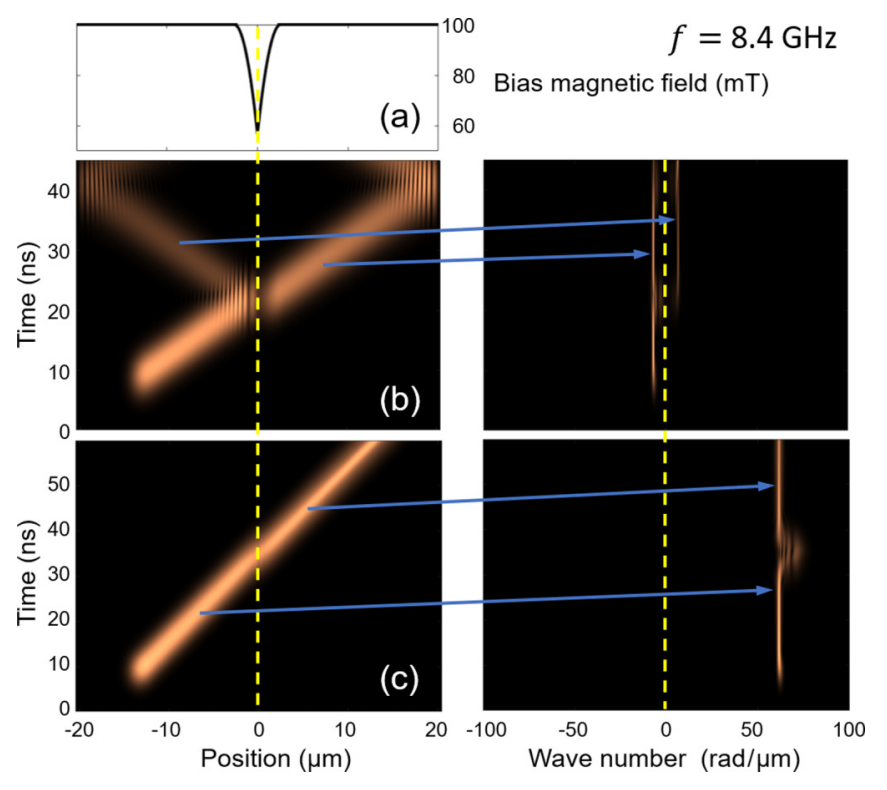

FIG. 5. The trajectories of a BVDESW wave packet passing through the bias magnetic field in (a) are shown in the real (left column) and reciprocal (right column) space for the frequency of 8.4 GHz. The brighter color corresponds to greater spin-wave amplitude. The initial values of the central wave number are $-6 \mathrm{rad} / \mu \mathrm{m}$ and $62 \mathrm{rad} / \mu \mathrm{m}$ in rows (b), (c), respectively. The arrows show correspondence between the real-space and reciprocal-space wave-packet envelopes. See the Supplemental Material [33] for an animated version of this figure.

ing from and transmission/tunneling through regions of local enhancement of the bias magnetic field.

Together, the possibility to create, to switch and to read out the pseudospin constitutes the backbone of the concept of magnon valleytronics. However, any more extensive investigation and exploitation of the concept goes beyond the scope of this paper.

\section{DISCUSSION}

The wavelength conversion occurring as a result of backscattering from magnetic-field increases mandates the use of the full version of the Bohr-Sommerfeld formula, Eq. (3), when employing the WKB approximation to describe confinement of BVDESWs. Hence, the analyses of mode confinement in spin-wave wells from Refs. [19-26], in which Eq. (4) was used, need to be revisited, even if qualitatively. To illustrate the arising peculiarities, Fig. 6 shows the phase-space trajectories corresponding to the three cases of spin-wave confinement presented in Figs. 2-4, which we discuss in the following. In this discussion, we deliberately avoid using the just introduced notion of the BVDESW pseudospin: it is too new and could therefore create more confusion rather than clarify things. However, we note that the pseudospin is switched every time a phase-space trajectory in Fig. 6 crosses line $k=0$.

We begin from a symmetric spin-wave well, which is relevant, e.g., to the FMR force microscopy (FMRFM) measurements reported by Chia et al. in Ref. [26]. At each frequency $f_{n}$ constrained by the $U$ point and FMR values 


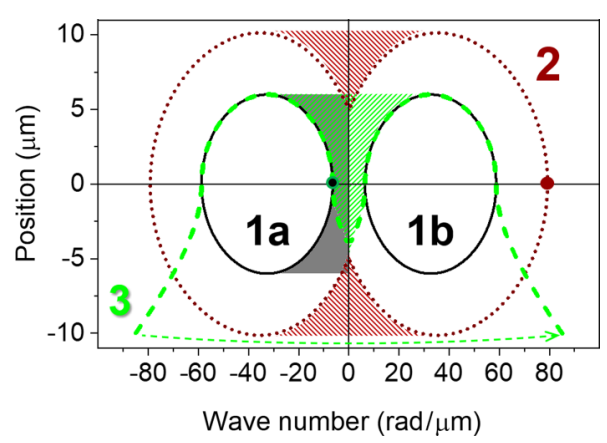

FIG. 6. The wave-packet trajectories for the field profile from Fig. 2 (curves 1 and 2) and its antisymmetric counterpart (curve 3) are shown in the phase space for the frequencies of $8.4 \mathrm{GHz}$ (curves $1 \mathrm{a}, 1 \mathrm{~b}$, and 3 ) and $10.5 \mathrm{GHz}$ (curve 2). The packets move counterclockwise starting from the points indicated by circles. The green dashed arrow shows the reciprocal-space jump of the packet due to its reflection from the edge at $-10.24 \mu \mathrm{m}$. The shaded areas show the difference between the accumulated phase calculated using Eqs. (3) and (4).

(at the field corresponding to the bottom of the well) and satisfying Eq. (3), there are two degenerate normal modes: one in each dispersion valley. The modes have oval-shaped phase-space trajectories, labeled "1a" and " $1 \mathrm{~b}$ " in Fig. 6. Each trajectory contains two reflections from field increases in the real space. In the reciprocal space, the reflections correspond to the same $U$ point albeit approached from opposite sides. Hence, the wavelength is converted twice and reaches the same value after one round trip in the well. Then, the quantization condition given by Eq. (3) corresponds to the constructive interference of the mode with itself, which occurs if the phase accumulated after the round trip is equal to $2 \pi n$. As the frequency reaches the FMR value from below, the two ovals touch at the $\Gamma$ point; at frequencies above the FMR value, only one mode, with an hourglasslike trajectory, labeled "2" in Fig. 6, remains. The trajectories at any frequency have a symmetry axis at $x=0$. At frequencies above the FMR value, line $k=0$ is also a symmetry axis.

The integration in the full Bohr-Sommerfeld formula [Eq. (3)] yields the accumulated phase as the area enclosed by a phase-space trajectory (Fig. 6). At the same time, the shortcut given by Eq. (4), a variant of which was used by Chia et al. [26], would additionally include the shaded areas. Hence, the use of Eq. (4) leads to an overestimation of the accumulated phase and therefore to an underestimation of the mode frequencies. This is likely to be one of the factors contributing to the failure of the WKB approximation to explain the FMRFM results in Ref. [26], with the confinement in the orthogonal in-plane direction and the intermodal coupling being others $[26,46]$.

For the antisymmetric well [19-24], the phase-space trajectory, labeled "3" in Fig. 6, has the only symmetry axis at $k=0$. During the first real-space round trip across the well, the wave is reflected once from the high-field turning point, which corresponds to the negative $U$ point in the reciprocal space, and once from the sample edge, which induces a reciprocal-space jump from $-k$ to $+k$. Therefore, the wavelength conversion occurs only once as a result of this round trip. Hence, the two copropagating waves at $x=0$ have different wavelengths and cannot amplify or cancel each other, regardless of their relative phase. The interference is reenabled after the second real-space round trip, which adds another wavelength converting reflection from the high-field turning point (now corresponding to the other, positive $U$ point) and a wavelength preserving reflection from the field decrease (corresponding to the $\Gamma$ point in the reciprocal space). As for the symmetric well, the accumulated phase yielded by the full Bohr-Sommerfeld formula [Eq. (3)] is equal to the area enclosed by the trajectory but is overestimated by Eq. (4), used in Refs. [19-24], by the amount equal to the shaded area. This could explain the need for additional phase jumps introduced but not explained in Ref. [23]. It is also clear that non-WKB-based analytical models put forward, e.g., in Refs. [24,46-49] to describe spin-wave confinement need to be revised to account for the behavior revealed here.

The Möbius-like and valley-confined wave-packet trajectories shown in Fig. 6 cannot be resolved explicitly for spin-wave wells with sizes below that of the BVDESW wave packet, although they should continue to have effect on the mode quantization and spatial character. We speculate that one piece of evidence for formation of Möbius and valleyconfined modes could be found in the character of their amplitude and phase profiles, respectively. Indeed, the amplitude profile of a standard standing mode contains nodes between antinodes, with nodes separating regions with phase differing by $\pi$. The traveling profiles inherent to valleyconfined modes should lead to a continuous rather than abrupt variation of phase. The very different wavelengths inherent to Möbius modes during different passages of the same regions should lead to a nonmonotonic amplitude variation (without nodes) within regions of the same or slowly varying phase. However, these effects may be obscured by the Schlömann emission [17] of spin waves from magnonic index nonuniformities [50] and from sample boundaries [51]. Hence, we leave the more detailed analysis of submicrometer spin-wave wells to future studies.

\section{CONCLUSIONS AND OUTLOOK}

We conclude by outlining other research directions that are likely to be affected by our main findings (the BVDESW wavelength conversion and Möbius mode formation) and by giving some further remarks on our observations.

(i) Our considerations should apply to other magnonic systems, e.g., van der Waals heterostructures discussed in the context of magnon valleytronics [52-54]; vice versa, some of the effects from Refs. [52-54] could be transferred to BVDESWs.

(ii) The spin-wave wavelength conversion (albeit probably not the Möbius mode formation) should occur in magnonic systems featuring a single valley at a finite wave number, with the shift induced, e.g., by the Dzyaloshinskii-Moriya interaction (DMI) interaction [55] or electric field [56].

(iii) The magnonic valley modes with different pseudospins form two different Bose-Einstein condensates [57], which can be spatially separated by a transient graded magnetic field [58]. Our results suggest that a local transient field 
decrease could induce mixing of the condensates of opposite pseudospin.

(iv) The wave amplitude increase (seen as brightening of the real-space trajectories in Figs. 2-4) near the high-field turning points is due to the wave shoaling effect (i.e., energy concentration due to reduction of the group velocity), as discussed earlier for other types of spin waves [59,60].

(v) The real-space relative shift between the trajectories of the incident and tunneled wave packets is a manifestation of the Hartman effect [61], demonstrated here for dipoleexchange spin waves. Its discussion for purely exchange spin waves can be found in Ref. [62].

(vi) A promising avenue for further research lies in combining spatial magnetic gradients, e.g., such as those considered here, with magnetic transients of the kinds considered in Refs. [63-66].

(vii) Our simulations are performed for graded 1D profiles of the bias magnetic field. However, Maxwell equations impose certain limitations on the feasibility of the profile's exact implementation [67]. Moreover, the assumed field vari- ation of $0.1 \mathrm{~T}$ over $10 \mu \mathrm{m}$ length scales is also quantitatively challenging to implement, although the strong demagnetizing fields in patterned magnetic structures $[19,20]$ and the stray field from the magnetic tip in FMRFM [26] suggest realistic ways forward. Yet, as with spin-wave Bloch oscillations [67], the effects described here are not restricted to graded magnetic fields [68] but could be generalized to other magnonic media with spatially varying characteristics, produced using the toolbox of graded index magnonics [69,70]. For instance, we note the two recent papers, which appeared while our paper was in review, studying spin-wave nonreciprocity in media with graded exchange [71] and magnetization [72].

\section{ACKNOWLEDGMENT}

The research leading to these results has received funding from the Engineering and Physical Sciences Research Council (EPSRC) of the United Kingdom via Grant No. EP/T016574/1.
[1] U. Leonhardt and T. G. Philbin, Transformation optics and the geometry of light, Prog. Opt. 53, 69 (2009).

[2] L. D. Landau and E. M. Lifshitz, Quantum Mechanics: Non-Relativistic Theory (Butterworth-Heinemann, Amsterdam, 1977).

[3] F. R. Hope, M. J. Baird, and A. F. G. Wyatt, Quantum Evaporation from Liquid ${ }^{4} \mathrm{He}$ by Rotons, Phys. Rev. Lett. 52, 1528 (1984)

[4] I. N. Adamenko, K. E. Nemchenko, and I. V. Tanatarov, Transmission and reflection of phonons and rotons at the superfluid helium-solid interface, Phys. Rev. B 77, 174510 (2008).

[5] Y. Castin, A. Sinatra, and H. Kurkjian, Landau Phonon-Roton Theory Revisited for Superfluid ${ }^{4} \mathrm{He}$ and Fermi Gases, Phys. Rev. Lett. 119, 260402 (2017).

[6] R. V. Vovk, C. D. H. Williams, and A. F. G. Wyatt, Some features of the long-pulse propagation mode of a phonon sheet in superfluid ${ }^{4}$ He, Low Temp. Phys. 44, 1062 (2018).

[7] A. Rycerz, J. Tworzydlo, and C. W. J. Beenakker, Valley filter and valley valve in graphene, Nat. Phys. 3, 172 (2007).

[8] A. V. Shytov, M. S. Rudner, and L. S. Levitov, Klein Backscattering and Fabry-Perot Interference in Graphene Heterojunctions, Phys. Rev. Lett. 101, 156804 (2008).

[9] A. H. Castro Neto, F. Guinea, N. M. R. Peres, K. S. Novoselov, and A. K. Geim, The electronic properties of graphene, Rev. Mod. Phys. 81, 109 (2009).

[10] C. A. Downing and M. E. Portnoi, Bielectron vortices in twodimensional Dirac semimetals, Nat. Commun. 8, 897 (2017).

[11] A. I. Akhiezer, V. G. Bar'yakhtar, and S. V. Peletminskii, Spin Waves (North-Holland, Amsterdam, 1968).

[12] A. G. Gurevich and G. A. Melkov, Magnetization Oscillations and Waves (Chemical Rubber Corp., New York, 1996).

[13] V. V. Kruglyak, S. O. Demokritov, and D. Grundler, Magnonics, J. Phys. D: Appl. Phys. 43, 264001 (2010).

[14] B. A. Kalinikos and A. N. Slavin, Theory of dipole-exchange spin wave spectrum for ferromagnetic films with mixed ex- change boundary conditions, J. Phys. C: Solid State Phys. 19, 7013 (1986).

[15] V. V. Kruglyak, P. S. Keatley, A. Neudert, M. Delchini, R. J. Hicken, J. R. Childress, and J. A. Katine, Imaging small-amplitude magnetization dynamics in a longitudinally magnetized microwire, Phys. Rev. B 77, 172407 (2008).

[16] J. Förster, J. Gräfe, J. Bailey, S. Finizio, N. Träger, F. Groß, S. Mayr, H. Stoll, C. Dubs, O. Surzhenko, N. Liebing, G. Woltersdorf, J. Raabe, M. Weigand, G. Schütz, and S. Wintz, Direct observation of coherent magnons with suboptical wavelengths in a single-crystalline ferrimagnetic insulator, Phys. Rev. B 100, 214416 (2019).

[17] E. Schlömann, Generation of spin waves in nonuniform magnetic field. I. Conversion of electromagnetic power into spin-wave power and vice versa, J. Appl. Phys. 35, 159 (1964).

[18] A. V. Vashkovskii and E. G. Lokk, Characteristics of a magnetostatic surface wave in a ferrite-dielectric structure embedded in a slowly varying nonuniform magnetic field, J. Commun. Technol. Electron. 46, 1163 (2001).

[19] J. Jorzick, S. O. Demokritov, B. Hillebrands, M. Bailleul, C. Fermon, K. Y. Guslienko, A. N. Slavin, D. V. Berkov, and N. L. Gorn, Spin Wave Wells in Nonellipsoidal Micrometer Size Magnetic Elements, Phys. Rev. Lett. 88, 047204 (2002).

[20] J. P. Park, P. Eames, D. M. Engebretson, J. Berezovsky, and P. A. Crowell, Spatially Resolved Dynamics of Localized SpinWave Modes in Ferromagnetic Wires, Phys. Rev. Lett. 89, 277201 (2002).

[21] K. Y. Guslienko, R. W. Chantrell, and A. N. Slavin, Dipolar localization of quantized spin-wave modes in thin rectangular magnetic elements, Phys. Rev. B 68, 024422 (2003).

[22] S. O. Demokritov, Dynamic eigen-modes in magnetic stripes and dots, J. Phys.: Condens. Matter 15, S2575 (2003).

[23] C. Bayer, S. O. Demokritov, B. Hillebrands, and A. N. Slavin, Spin-wave wells with multiple states created in small magnetic elements, Appl. Phys. Lett. 82, 607 (2003). 
[24] C. Bayer, J. P. Park, H. Wang, M. Yan, C. E. Campbell, and P. A. Crowell, Spin waves in an inhomogeneously magnetized stripe, Phys. Rev. B 69, 134401 (2004).

[25] M. P. Kostylev, A. A. Serga, T. Schneider, T. Neumann, B. Leven, B. Hillebrands, and R. L. Stamps, Resonant and nonresonant scattering of dipole-dominated spin waves from a region of inhomogeneous magnetic field in a ferromagnetic film, Phys. Rev. B 76, 184419 (2007).

[26] H.-J. Chia, F. Guo, L. M. Belova, and R. D. McMichael, Nanoscale Spin Wave Localization Using Ferromagnetic Resonance Force Microscopy, Phys. Rev. Lett. 108, 087206 (2012).

[27] K. R. Smith, M. J. Kabatek, P. Krivosik, and M. Wu, Spin wave propagation in spatially nonuniform magnetic fields, J. Appl. Phys. 104, 043911 (2008).

[28] J. Leliaert, M. Dvornik, J. Mulkers, J. De Clercq, M. V. Milosevic, and B. Van Waeyenberge, Fast micromagnetic simulations on GPU-recent advances made with MuMax3, J. Phys. D: Appl. Phys. 51, 123002 (2018).

[29] M. Dvornik, Y. Au, and V. V. Kruglyak, Micromagnetic simulations in magnonics, Top. Appl. Phys. 125, 101 (2013).

[30] N. J. Whitehead, S. A. R. Horsley, T. G. Philbin, and V. V. Kruglyak, A Luneburg lens for spin waves, Appl. Phys. Lett. 113, 212404 (2018).

[31] N. J. Whitehead, S. A. R. Horsley, T. G. Philbin, and V. V. Kruglyak, Graded index lenses for spin wave steering, Phys. Rev. B 100, 094404 (2019).

[32] S.-K. Kim, S. Choi, K.-S. Lee, D.-S. Han, D.-E. Jung, and Y.-S. Choi, Negative refraction of dipole-exchange spin waves through a magnetic twin interface in restricted geometry, Appl. Phys. Lett. 92, 212501 (2008).

[33] See Supplemental Material at http://link.aps.org/supplemental/ 10.1103/PhysRevB.103.184403 for animated versions of Figs. 2-5.

[34] D. A. Bozhko, H. Y. Musiienko-Shmarova, V. S. Tiberkevich, A. N. Slavin, I. I. Syvorotka, B. Hillebrands, and A. A. Serga, Unconventional spin currents in magnetic films, Phys. Rev. Res. 2, 023324 (2020).

[35] P. M. Gunnink, R. A. Duine, and A. Rückriegel, Electrical detection of unconventional transverse spin currents in obliquely magnetized thin films, Phys. Rev. B 101, 220407 (2020).

[36] S. O. Demokritov, A. A. Serga, A. André, V. E. Demidov, M. P. Kostylev, B. Hillebrands, and A. N. Slavin, Tunneling of Dipolar Spin Waves through a Region of Inhomogeneous Magnetic Field, Phys. Rev. Lett. 93, 047201 (2004).

[37] U.-H. Hansen, M. Gatzen, V. E. Demidov, and S. O. Demokritov, Resonant Tunneling of Spin-Wave Packets via Quantized States in Potential Wells, Phys. Rev. Lett. 99, 127204 (2007).

[38] U.-H. Hansen, V. E. Demidov, and S. O. Demokritov, Dualfunction phase shifter for spin-wave logic applications, Appl. Phys. Lett. 94, 252502 (2009).

[39] T. Neumann, A. A. Serga, B. Hillebrands, and M. P. Kostylev, Frequency-dependent reflection of spin waves from a magnetic inhomogeneity induced by a surface direct current, Appl. Phys. Lett. 94, 042503 (2009).

[40] A. A. Serga, T. Neumann, A. V. Chumak, and B. Hillebrands, Generation of spin-wave pulse trains by current-controlled magnetic mirrors, Appl. Phys. Lett. 94, 112501 (2009).
[41] M. Mohseni, B. Hillebrands, P. Pirro, and M. Kostylev, Controlling the propagation of dipole-exchange spin waves using local inhomogeneity of the anisotropy, Phys. Rev. B 102, 014445 (2020).

[42] S. O. Demokritov, A. A. Serga, V. E. Demidov, B. Hillebrands, M. P. Kostylev, and B. A. Kalinikos, Experimental observation of symmetry-breaking nonlinear modes in an active ring, Nature 426, 159 (2003).

[43] Y. Au, E. Ahmad, O. Dmytriiev, M. Dvornik, T. Davison, and V. V. Kruglyak, Resonant microwave-to-spin-wave transducer, Appl. Phys. Lett. 100, 182404 (2012).

[44] Y. Au, M. Dvornik, O. Dmytriiev, and V. V. Kruglyak, Nanoscale spin wave valve and phase shifter, Appl. Phys. Lett. 100, 172408 (2012).

[45] V. V. Kruglyak, C. S. Davies, Y. Au, F. B. Mushenok, G. Hrkac, N. J. Whitehead, S. A. R. Horsley, T. G. Philbin, V. D. Poimanov, R. Dost, D. A. Allwood, B. J. Inkson, and A. N. Kuchko, Graded magnonic index and spin wave fano resonances in magnetic structures: Excite, direct, capture, in Spin Wave Confinement. Propagating Waves, edited by S. O. Demokritov (Jenny Stanford Publishing, New York, 2017), Chap. 1.

[46] E. V. Tartakovskaya, M. Pardavi-Horvath, and R. D. McMichael, Spin-wave localization in tangentially magnetized films, Phys. Rev. B 93, 214436 (2016).

[47] G. Gubbiotti, M. Conti, G. Carlotti, P. Candeloro, E. Di Fabrizio, K. Y. Guslienko, A. Andre, C. Bayer, and A. N. Slavin, Magnetic field dependence of quantized and localized spin wave modes in thin rectangular magnetic dots, J. Phys. Condens. Matter 16, 7709 (2004).

[48] M. Bailleul, R. Höllinger, and C. Fermon, Microwave spectrum of square Permalloy dots: Quasisaturated state, Phys. Rev. B 73, 104424 (2006).

[49] Z. Duan, I. N. Krivorotov, R. E. Arias, N. Reckers, S. Stienen, and J. Lindner, Spin wave eigenmodes in transversely magnetized thin film ferromagnetic wires, Phys. Rev. B 92, 104424 (2015).

[50] C. S. Davies, V. D. Poimanov, and V. V. Kruglyak, Mapping the magnonic landscape in patterned magnetic structures, Phys. Rev. B 96, 094430 (2017).

[51] F. B. Mushenok, R. Dost, C. S. Davies, D. A. Allwood, B. Inkson, G. Hrkac, and V. V. Kruglyak, Broadband conversion of microwaves into propagating spin waves in patterned magnetic structures, Appl. Phys. Lett. 111, 042404 (2017).

[52] R. Hidalgo-Sacoto, R. I. Gonzalez, E. E. Vogel, S. Allende, J. D. Mella, C. Cardenas, R. E. Troncoso, and F. Munoz, Magnon valley Hall effect in $\mathrm{CrI}_{3}$-based van der Waals heterostructures, Phys. Rev. B 101, 205425 (2020).

[53] X. Zhai and Y. M. Blanter, Topological valley transport of gapped Dirac magnons in bilayer ferromagnetic insulators, Phys. Rev. B 102, 075407 (2020).

[54] D. Ghader, Valley-polarized domain wall magnons in 2D ferromagnetic bilayers, Sci. Rep. 10, 16733 (2020).

[55] B. W. Zingsem, M. Farle, R. L. Stamps, and R. E. Camley, Unusual nature of confined modes in a chiral system: Directional transport in standing waves, Phys. Rev. B 99, 214429 (2019).

[56] V. N. Krivoruchko, A. S. Savchenko, and V. V. Kruglyak, Electric-field control of spin-wave power flow and caustics in thin magnetic films, Phys. Rev. B 98, 024427 (2018). 
[57] P. Nowik-Boltyk, O. Dzyapko, V. E. Demidov, N. G. Berloff, and S. O. Demokritov, Spatially non-uniform ground state and quantized vortices in a two-component Bose-Einstein condensate of magnons, Sci. Rep. 2, 482 (2012).

[58] I. V. Borisenko, V. E. Demidov, V. L. Pokrovsky, and S. O. Demokritov, Spatial separation of degenerate components of magnon Bose-Einstein condensate by using a local acceleration potential, Sci. Rep. 10, 14881 (2020).

[59] O. Kolokoltsev, I. Gómez-Arista, N. Qureshi, A. Acevedo, C. L. Ordóñez-Romero, and A. Grishin, Compression gain of spin wave signals in a magnonic YIG waveguide with thermal nonuniformity, J. Magn. Magn. Mater. 377, 1 (2015).

[60] N. Perez and L. Lopez-Diaz, Magnetic field induced spin-wave energy focusing, Phys. Rev. B 92, 014408 (2015).

[61] T. E. Hartman, Tunneling of a wave packet, J. Appl. Phys. 33, 3427 (1962).

[62] J. W. Kłos, Y. S. Dadoenkova, J. Rychły, N. N. Dadoenkova, I. L. Lyubchanskii, and J. Barnaś, Hartman effect for spin waves in exchange regime, Sci. Rep. 8, 17944 (2018).

[63] S. M. Rezende and F. R. Morgenthaler, Magnetoelastic waves in time-varying magnetic fields. I. Theory, J. Appl. Phys. 40, 524 (1969).

[64] S. M. Rezende and F. R. Morgenthaler, Magnetoelastic waves in time-varying magnetic fields. II. Experiments, J. Appl. Phys. 40, 537 (1969).
[65] V. L. Preobrazhenskii and Y. K. Fetisov, Magnetostatic waves in a time-dependent medium, Sov. Phys. J. 31, 898 (1988).

[66] J.-N. Toedt and W. Hansen, Dynamic control of spin-wave propagation, Sci. Rep. 11, 7821 (2021).

[67] A. S. Laurenson, J. Bertolotti, and V. V. Kruglyak, Bloch oscillations of backward volume magnetostatic spin waves, Phys. Rev. B 102, 054416 (2020).

[68] V. N. Samofalov, D. P. Belozorov, and A. G. Ravlik, Strong stray fields in systems of giant magnetic anisotropy magnets, Phys.-Usp. 56, 269 (2013).

[69] C. S. Davies and V. V. Kruglyak, Graded-index magnonics, Low Temp. Phys. 41, 760 (2015).

[70] S. Mieszczak, O. Busel, P. Gruszecki, A. N. Kuchko, J. W. Kłos, and M. Krawczyk, Anomalous Refraction of Spin Waves as a Way to Guide Signals in Curved Magnonic Multimode Waveguides, Phys. Rev. Appl. 13, 054038 (2020).

[71] R. Macêdo, A. S. Kudinoor, K. L. Livesey, and R. E. Camley, Breaking spacial inversion-symmetry to obtain nonreciprocal spin-wave excitation: The case of nonuniform magnetic exchange, arXiv:2012.10381.

[72] P. Borys, O. Kolokoltsev, N. Qureshi, M. L. Plumer, and T. L. Monchesky, Unidirectional spin wave propagation due to a saturation magnetization gradient, Phys. Rev. B 103, 144411 (2021). 